\title{
Integridade da pele prejudicada em idosos: estudo de ocorrência numa comunidade atendida pelo Programa Saúde da Família
}

\author{
Impaired skin integrity in the elderly: study of incidence from a community attending a Family \\ Health Program \\ Integridad de la piel perjudicada en ancianos: estudio de incidencia en una comunidad atendida por \\ el Programa Salud de la Família
}

\section{Daniella de Moura Resende ${ }^{1}$, Maria Márcia Bachion², Lorena Aparecida de Oliveira Araújo ${ }^{3}$}

\section{RESUMO}

Objetivo: esta pesquisa teve como objetivo estudar a ocorrência de integridade da pele prejudicada em idosos de uma comunidade atendida pelo Programa Saúde da Família. Métodos: participaram deste estudo descritivo 40 idosos. A coleta de dados ocorreu de janeiro a junho de 2004 e constou de entrevista e exame clínico. Resultados: A integridade da pele prejudicada foi encontrada em 20 idosos (50\%). A característica definidora de maior freqüência foi rompimento da superfície da pele ( $85 \%$ ). Os fatores relacionados identificados com freqüência maior ou igual a $50 \%$ foram: extremos de idade (100\%), fatores imunológicos (100\%), alteração do turgor (100\%), fatores mecânicos (85\%), medicação em uso (50\%). Conclusão: a integridade de pele prejudicada atingiu metade dos sujeitos desta pesquisa, indicando necessidade de intervenções de enfermagem para prevenção e recuperação de lesões, além de promoção da saúde, buscando a integridade tegumentar.

Descritores: Idoso; Diagnóstico de enfermagem; Programa saúde da família; Enfermagem geriátrica

\begin{abstract}
Objective: this descriptive study has the general objective of studying the occurrence of impaired skin integrity in the elderly from a community attending a Family Health Program in Goiânia, Brazil. Methods: forty elders participated in this study. The data collection occurred from January until June at 2004, at the participants' residences, where we conducted interviews and clinical exams. Results: the skin integrity impaired was found in 20 participants (50\%). The defining characteristic of a higher frequency was the disruption of the skin surface $(85 \%)$. The related factors identified with a higher or equal frequency of 50\% were: age extremes $(100 \%)$, immunological factors $(100 \%)$, alteration in turgor $(100 \%)$, mechanical factors $(85 \%)$, usual medication $(50 \%)$. Conclusion: these results point to the necessity of nursing interventions for prevention and recovery of lesions, besides promotion of the health as a whole, looking for the skin integrity. Keywords: Aged; Nursing diagnosis; Health family program; Geriatric nursing
\end{abstract}

\section{RESUMEN}

Objetivo: esta investigación tuvo como objetivo estudiar la ocurrencia de integridad de la piel perjudicada en ancianos de una comunidad atendida por el Programa Salud de la Familia. Método: participaron de este estudio descriptivo 40 ancianos. La recolección de los datos ocurrió de enero a junio del 2004 y constó de entrevista y examen clínico. Discusión: la integridad de la piel perjudicada fue encontrada en 20 ancianos (50\%). La característica que definió la mayor frecuencia fue la ruptura de la superficie de la piel (85\%). Los factores relacionados identificados con mayor o igual frecuencia a 50\% fueron: extremos de edad (100\%), factores inmunológicos (100\%), alteración de la turgencia $(100 \%)$, factores mecánicos (85\%), medicación en uso (50\%). Conclusión: la integridad de la piel perjudicada alcanzó a la mitad de los sujetos de esta investigación, indicando la necesidad de intervenciones de enfermería para la prevención y recuperación de las lesiones, además de la promoción de la salud, buscando la integridad tegumentaria.

Descriptores: Anciano; Diagnóstico de enfermería; Programa salud de la familia; Enfermería geriátrica

\footnotetext{
1 Acadêmica da Faculdade de Enfermagem da Universidade Federal de Goiás FEN-UFG-Goiânia (GO), Brasil.

${ }^{2}$ Doutora em Enfermagem. Professora Titular da FEN - UFG - Goiânia (GO), Brasil..

${ }^{3}$ Mestre em Ciências da Saúde. Secretaria Municipal de Saúde de Goiânia. Programa Saúde da Família - Goiânia (GO), Brasil.
} 


\section{INTRODUÇÃO}

O envelhecimento não é conceituado como doença, mas pode determinar um grande número de transtornos. Um dos sinais mais evidentes da passagem dos anos está relacionado com as mudanças produzidas no aspecto da pele, caracterizadas por alterações na sua estrutura e funções ${ }^{(1-3)}$.

Estudos mostram que a pele do adulto sofre diversas e profundas transformações com o avanço da idade. Entre as alterações fisiológicas do tecido tegumentar que estão presentes no idoso destacam-se maior fragilidade cutânea e menor capacidade da pele de atuar como barreira contra fatores externos; termorregulação deficiente em resposta ao calor, decorrente da diminuição do número de glândulas sudoríparas; pele mais seca e rugosa por causa do menor número de glândulas sebáceas, resultando em pequena produção de óleo; menor estímulo sensitivo; diminuição da elasticidade, flacidez, alteração da resposta imunológica celular ${ }^{(4)} \mathrm{e}$ diminuição da espessura da derme e da epiderme ${ }^{(5)}$. O risco de lesão da pele aumenta em virtude da diminuição da função de barreira. Por outro lado, há maior dificuldade em perceber estímulos traumáticos e agressivos, devido a capacidade reduzida dos receptores sensoriais ${ }^{(2)}$.

A pele reflete condições físicas e psicológicas, como saúde, idade e diferenças étnicas e culturais, suas funções incluem a proteção, a excreção, regulação da temperatura, percepção sensitiva e imagem corporal. Assim, manter a pele íntegra é fundamental para que o organismo possa se defender contra alterações diversas, traumatismo mecânicos, térmicos, químicos, radiação ${ }^{(1-2)}$, ou situações múltiplas a que o ser humano está exposto durante toda sua vida.

A enfermagem precisa produzir conhecimentos que aprimorem o cuidado junto ao idoso. Ao nomear condições que requerem intervenções de enfermagem, estabelecemos diagnósticos de enfermagem. Para rotulálos de modo uniforme, dispomos de várias taxonomias de classificação de diagnósticos. A mais utilizada em nosso país é a Taxonomia da North American Nursing Diagnosis Association (NANDA), que inclui título diagnóstico, definição, características definidoras (pistas ou evidências, sinais e sintomas), fatores de risco (fatores que aumentam a vulnerabilidade ao evento) e fatores relacionados (etiologia, fator coadjuvante ou antecedente). Os diagnósticos de enfermagem podem ser reais, de risco ou de bem estar( ${ }^{(*)}$.

De acordo com esta taxonomia, a Integridade da pele prejudicada é um diagnóstico real, que se refere à "epiderme e/ou derme alteradas", devido a fatores externos, que incluem: hipertermia ou hipotermia, substância química, umidade, fatores mecânicos (por exemplo, adesivos que arrancam os pêlos, pressão, contensão), imobilização física, radiação, idades extremas, pele molhada, medicações e fatores internos, tais como, estado metabólico alterado, proeminências esqueléticas, déficit imunológico, fatores de desenvolvimento, sensibilidade alterada, estado nutricional alterado (por exemplo, obesidade, emagrecimento), pigmentação alterada, circulação alterada, alterações no turgor (mudanças na elasticidade), estado dos líquidos alterados. São consideradas pistas (características definidoras) para esta ocorrência a "invasão de estruturas do corpo, destruição de camadas da pele (derme), rompimento da superfície da pele (epiderme)"(()).

Apesar de a literatura afirmar que problemas relativos à integridade da pele são esperados nos idosos ${ }^{(7-8)}$, dispomos de poucos estudos que tenham gerado informações precisas quanto à incidência e prevalência de agravos nesse sentido, na população idosa na comunidade.

Frente ao exposto, propusemos a realização do presente estudo com a finalidade de produzir informações que pudessem subsidiar políticas de atenção à saúde desta população, com vistas à detecção precoce de agravos e promoção da saúde, visto que a população idosa é susceptível a alterações na integridade da pele e, com isso, pode sofrer prejuízos na qualidade de vida.

\section{OBJETIVOS}

O objetivo geral desta pesquisa foi estudar a ocorrência do diagnóstico de enfermagem integridade da pele prejudicada em idosos de uma comunidade.

Nesse sentido, foram estabelecidos como objetivos específicos verificar a incidência de integridade da pele prejudicada em idosos de uma comunidade em Goiânia, de acordo com o sexo e a faixa etária; identificar a freqüência das características definidoras e dos fatores relacionados, bem como analisar as características definidoras e os fatores relacionados para este diagnóstico nesta população.

\section{MÉTODOS}

Trata-se de um estudo descritivo, que envolveu a população de idosos (60 anos ou mais) moradores do Jardim Mariliza em Goiânia (Região/ Distrito Sul), atendidos pela equipe 02 do Programa Saúde da Familia. A amostra foi constituída por 40 idosos que se dispuseram a participar do estudo, entre os 102 cadastrados na área. A escolha desta região foi realizada em função da inserção prévia das pesquisadoras na área e da colaboração já consolidada dos integrantes desta equipe em pesquisas anteriormente realizadas.

O Projeto de pesquisa obteve parecer favorável da 
Coordenação Colegiada do Programa Saúde da Família da Secretaria de Saúde de Goiânia, do Conselho Diretor da Faculdade de Enfermagem da Universidade Federal de Goiás (UFG) e do Comitê de Ética em Pesquisa da UFG.

Acessamos a listagem dos idosos cadastrados no PSF do Jardim Mariliza com a finalidade de agendar visita domiciliária para convidar os moradores com 60 anos ou mais para participar do estudo, mediante consentimento livre e esclarecido por escrito, sendo garantido sigilo e anonimato aos mesmos.

Buscamos contatar (com ajuda dos agentes comunitários da região) todos os idosos moradores da área. Deixaram de ser incluídos aqueles que não foram encontrados no domicílio e os que se recusaram a participar. Após aceite, foram agendados data e local para coleta de dados que foi realizada no período de janeiro a junho de 2004.

O instrumento de coleta de dados foi elaborado tendo como referencial a revisão da literatura e a Taxonomia da NANDA ${ }^{(6)}$. Este roteiro foi analisado por dois enfermeiros com experiência no atendimento a idosos, que avaliaram a pertinência, abrangência e organização do protocolo, que incluiu um check list dos fatores que podem levar ao comprometimento da integridade da pele e evidências de integridade da pele prejudicada. Em seu formato final o roteiro continha apenas características definidoras contidas na NANDA ${ }^{(6)}$, uma vez que nesse processo de refinamento não foram encontradas novas evidências, além daquelas já incluídas na referida taxonomia.

O procedimento de coleta de dados ocorreu em ambiente privativo, no domićlíio do idoso ou no consultório de enfermagem do PSF do setor, como o idoso preferiu. Realizamos procedimentos de entrevista e exame clínico, que incluíram a observação e a mensuração, além de teste de sensibilidade tátil (com monofilamento de nylon).

Em um segundo momento, mediante processo de análise das informações disponíveis na avaliação dos participantes, os dados foram comparados com a taxonomia da $\operatorname{NANDA}^{(6)}$, e então foi elaborado julgamento clínico da presença ou não dos fatores relacionados, características definidoras, e, por fim, do diagnóstico em estudo. Para análise dos resultados obtidos, utilizamos procedimentos de estatística descritiva, a freqüência simples e medidas de tendência central.

\section{RESULTADOS}

Dos 40 idosos que participaram do estudo, 27 $(67,5 \%)$ eram do sexo feminino e $13(35,5 \%)$ do masculino. A idade dos mesmos variou de 60 a 86 anos, sendo a média igual a 70 anos. Quanto à escolaridade, $30(75 \%)$ eram alfabetizados, enquanto $10(25 \%)$ eram analfabetos.

Foi identificada integridade da pele prejudicada em $50 \%$ dos sujeitos. A distribuição deste evento ocorreu de forma semelhante, comparando-se a proporção de homens e mulheres. A integridade da pele prejudicada esteve presente em $51,85 \%$ das 27 mulheres e $46,15 \%$ dos 13 homens.

Quanto à distribuição proporcional por faixa etária, encontramos que entre idosos de 60-69 anos, 57,14\% apresentaram este diagnóstico, entre os idosos na faixa de 70 a 79 anos, $38,46 \%$ e entre os maiores de 80 anos, a ocorrência foi de $50 \%$.

Prosseguindo, apresentaremos os achados em relação às características definidoras e fatores relacionados, tomando como referência os 20 idosos que apresentaram integridade da pele prejudicada.

Identificamos nos participantes as seguintes características definidoras para este diagnóstico: rompimento da superfície da pele (epiderme) em 17 idosos (85\%); destruição de camadas da pele (derme) verificada em 11 idosos (55\%) e invasão de estruturas do corpo, presente em 8 idosos (40\%).

Observamos em todos os idosos que fizeram parte da amostra a presença de manchas ou máculas que são alterações cutâneas circunscritas, caracterizadas apenas pela modificação da cor natural da pele ${ }^{(9)}$. Da mesma forma, foi notável a presença de idosos com manchas senis ou melanose senil, em que os melanócitos diminuem em número e sofrem alterações funcionais, acarretando o aparecimento de manchas hipercrômicas, planas e lisas, principalmente na face e no dorso da mão. Pelo fato dessas manchas não constituírem modificações na integridade da superfície da pele ou de suas camadas subjacentes ${ }^{(10)}$, elas não foram consideradas como característica definidora para esse diagnóstico.

A característica definidora de maior freqüência foi o rompimento de superfície da pele (epiderme) evidenciada pela presença de lesões, como escoriações, regiões eritematosas, descamações, com tamanhos variando entre 0,5 e $4 \mathrm{~cm}$ de diâmetro para lesões arredondadas e até $10 \mathrm{~cm}$ para lesões lineares. A maioria se apresentava limpa e em processo final de cicatrização (formação de crosta e fase de epitelização); algumas mostravam a derme ainda alterada.

Não foram encontradas feridas de grande extensão. Apenas um idoso apresentou ferida crônica no membro inferior direito, com diâmetro de aproximadamente 4 $\mathrm{cm}$, profundidade de $1 \mathrm{~cm}$, presença de sinais flogísticos, cicatrização lenta e difícil. Apresentava-se contaminada, com exsudato seroso, hemorrágico, supurativo e inodoro. Esta ferida se configurava como úlcera venosa de estase. 
Nesta amostra não foram encontradas escaras, úlceras arteriais ou neuropáticas, como esperado, levando em conta as informações disponíveis em outros estudos realizados ${ }^{(7-8)}$.

Com relação aos 19 fatores relacionados previstos para integridade de pele prejudicada, os participantes apresentaram de 2 a 9 destes fatores, sendo a média de fatores por idoso igual a 4,25, a moda igual a 2 , o primeiro quartil igual a 2 e o terceiro quartil igual a 5 . A mediana correspondeu a 4 fatores relacionados.

Alguns fatores relacionados encontrados nos idosos apresentam-se inter-relacionados, tais como extremos de idade $(100 \%)$, alterações no turgor da pele $(100 \%)$ e fatores imunológicos (100\%), como apresentado na Tabela 1. Tais fatores, assim como outros que serão descritos posteriormente, foram interpretados como fatores coadjuvantes ou contribuintes, uma vez que isoladamente, não produzem perda de integridade na pele.

Tabela 1 - Distribuição dos fatores relacionados de integridade da pele prejudicada em idosos de uma comunidade de Goiânia (GO) Jan/Jun 2004

\begin{tabular}{lll}
\hline Fatores Relacionados & $f$ & $\%$ \\
\hline Idades extremas & 20 & 100 \\
Alteração no turgor (mudança na elasticidade) & 20 & 100 \\
Déficit imunológico & 20 & 100 \\
Fatores mecânicos & 17 & 85 \\
Medicações em uso & 10 & 50 \\
Estado de líquidos alterados & 9 & 45 \\
Pele contra-se hipertérmica & 4 & 20 \\
Estado metabólico alterado & 4 & 20 \\
Fatores de desenvolvimento & 4 & 20 \\
Circulação alterada & 3 & 15 \\
Exposição aumentada à radiação & 1 & 5 \\
Proeminências esqueléticas & 1 & 5 \\
Sensibilidade alterada (para menos) & 1 & 5 \\
Estado nutricional alterado (obesidade, & 1 & 5 \\
emagrecimento) & & \\
\hline
\end{tabular}

Além da perda de imunidade fisiológica, dos 20 idosos, cinco $(25 \%)$ apresentaram doenças que comprometem a função imunológica (diabetes e hipotireoidismo), ou faziam uso de medicação (psicotrópico) que deprimem o sistema imune e propiciam a ocorrência de lesões ou fazem com que a cicatrização dessas lesões seja retardada.

As mudanças na elasticidade e alterações no turgor da pele foram claramente percebidas nos 20 participantes $(100 \%)$. Normalmente, no idoso qualquer choque mais forte ou abrupto é potencialmente capaz de promover ruptura da pele, prejudicando sua inteireza, já que as fibras de elastina e de colágeno diminuem, gerando menor flexibilidade, elasticidade e força tênsil ${ }^{(2)}$.
Essa elasticidade diminuída, por sua vez, está diretamente associada ao volume de líquido que é ingerido durante as 24 horas do dia. Observamos em nove idosos (45\%) ingestão abaixo da quantidade ideal para a manutenção da homeostasia, que é de dois litros por dia. Os participantes referiram ingerir aproximadamente um litro de líquidos por dia. Ambos os fatores foram interpretados como coadjuvantes.

$\mathrm{O}$ fator relacionado que mais diretamente interferiu no aparecimento da integridade da pele prejudicada foi o trauma mecânico (85\%). Nesta categoria estão incluídos: pressão (trauma mecânico tipo esbarrão, 7 idosos), ferimentos com faca ou fogo (durante a preparação dos alimentos - 4 idosos), atrito ao caminhar ou correr em terreno áspero descalço (1 idoso), e traumas ocorridos em quedas (3 idosos), uso de prótese dentária mal adaptada (3 idosos).

Foi detectada em 6 idosos $(15 \%)$ a presença de circulação alterada sendo expressa pela insuficiência venosa percebida nas veias superficiais dos membros inferiores, juntamente com edema, eritrose, vermelhidão, cianose, hematomas e varizes.

A hipertermia na pele foi observada em $20 \%$ da amostra. Embora seja considerada como um fator relacionado para integridade da pele prejudicada, nesse caso, trata-se de uma reação inflamatória decorrente da lesão. Por outro lado, a redução da vascularização e do tecido subcutâneo, aliados à termorregulação deficiente da pele do idoso em resposta ao calor ${ }^{(10)}$, contribuem para a elevação da temperatura corporal dos mesmos, sem que necessariamente seja produzida alteração na integridade da pele.

O estado metabólico alterado, evidenciado em quatro idosos (20\%), ocorreu na vigência de doenças como diabetes e hipotireoidismo. Essas doenças conferem agravos que atingem potencialmente o tecido cutâneo, por isso são caracterizadas como fator causador de lesões ou agravante das já existentes ${ }^{(10)}$.

Em fatores ligados ao desenvolvimento, considerouse a dificuldade em equilibrar-se, que é característica nos extremos de idade. Esse fator foi observado em $20 \%$ dos participantes.

Em um participante (5\%), a exposição aumentada à radiação foi responsável pelo surgimento da lesão cutânea. Os idosos possuem maior sensibilidade e menor tolerância à exposição à radiação solar ${ }^{(2)}$. A exposição à radiação solar das 10 às 16 horas é um dos maiores responsáveis pelo câncer de pele. A lesão deste participante não se configurava em alteração desta natureza.

O estado nutricional alterado, evidenciado pelo emagrecimento foi observado em 1 idoso (5\%), que apresentava proeminências ósseas que se constituíram fator coadjuvante para aparecimento de lesão 
tegumentar.

A avaliação das sensibilidades dolorosa, tátil e térmica permitiu verificar que um idoso (5\%) apresentava menor sensibilidade. Os fatores relacionados capacidade de movimentação prejudicada, pele molhada, pigmentação alterada (para menos), substância química e pele hipotérmica não foram identificados entre os participantes.

\section{DISCUSSÃO}

A integridade de pele prejudicada entre idosos de 60 a 69 anos ocorre, possivelmente, pelo fato de que nesta idade os mesmos são mais ativos, em comparação com aqueles que apresentam idade mais avançada. Os idosos mais jovens têm maior contato com agentes agressores da integridade cutânea. O segundo grupo de maior ocorrência foi o de idosos com mais de 80 anos. $\mathrm{Na}$ idade mais avançada, os fatores de proteção fisiológicos podem estar com suas reservas já comprometidas, fazendo com que agentes agressivos em geral ganhem maior poder lesivo.

A idade avançada é acompanhada de alterações no turgor da pele e diminuição da função imunológica. No grupo estudado estes fatores são importantes coadjuvantes para a perda da integridade de pele. À medida que a pessoa envelhece, aumenta o risco de aparecerem lesões no tegumento, já que este se torna mais fino, mais frágil e ocorre perda na camada de gordura subcutânea. Além disso, há uma diminuição da sensibilidade ao tato, pressão, vibração, calor e frio, assim, um dos aspectos sistêmicos mais importantes, como cofator de risco tanto para a lesão como para a sua manutenção é a idade, ao gerar um profundo impacto no funcionamento de todos os sistemas fisiológicos corporais $^{(2)}$. A capacidade do corpo de manter a homeostasia torna-se progressivamente diminuída com o envelhecimento celular, já que ocorrem alterações mensuráveis no formato e na constituição $\operatorname{corporal}^{(3)}$. A pele torna-se mais vulnerável às irritações, menos tolerante a extremos de temperatura e exposição ao sol ${ }^{(3)}$, devido à flacidez, ressecamento, manchas e outras alterações vasculares e degenerativas. Essas e outras mudanças na integridade da pele estão relacionadas ainda à diminuição da elasticidade dérmica e à diminuição do funcionamento e do número das glândulas sebáceas e sudoríparas. Estas modificações comprometem a função de barreira, que impede a entrada de microorganismos, bem como a capacidade de recuperação às agressões de toda ordem ao nível da pele, tornando o idoso particularmente vulnerável a várias doenças de pele e a traumatismos diversos ${ }^{(10)}$.

Algumas mudanças na composição corporal são evidentes no processo de envelhecimento, tais como a redução do teor de água corporal, redução da massa corporal livre de gordura e modificações na distribuição da gordura corporal ${ }^{(2-3)}$. Quando essas alterações são exacerbadas, podem interferir em diversas outras funções corporais, tais como: função pulmonar, diminuição da imunidade, perda de força motora, assim, a imunidade celular e a humoral estão diminuídas. Em conseqüência disso, se instala no idoso uma menor resistência às infecções ${ }^{(10)}$, o que se deve, também, à diminuição da imunocompetência tissular, através da redução das células de Langerhans no tegumento ${ }^{(2)}$.

Os idosos apresentam maior tendência à instabilidade postural, alterações da marcha, que apresenta passos mais $\operatorname{curtos}^{(2)}$, menor velocidade de movimento, diminuição do fluxo sanguíneo cerebral, diminuição na velocidade de condução nervosa, menor tempo de reação, diminuição de tamanho e número de neurônios ${ }^{(3,10)}$. A musculatura torna-se mais frágil e atrofiada, as articulações tornam-se progressivamente enrijecidas e a quantidade de ossos saudáveis diminui ${ }^{2}$. De um modo geral, a estrutura e a função do sistema nervoso mudam com o avançar da idade o que acarreta um tempo de reação mais lento, levando o idoso a sofrer quedas e lesões ${ }^{(3)}$.

Outra mudança importante é a circulação alterada nos membros inferiores dos idosos, que são decorrentes principalmente da imobilidade e da diminuição do tônus muscular, conseqüente à atrofia senil ${ }^{(10)}$. Esta condição leva à suscetibilidade dos mesmos para apresentarem úlcera venosa de estase, associada principalmente com hipertensão $\operatorname{arterial}^{(7-8)}$.

Acrescenta-se, ainda, o fato de que, nesse grupo populacional, os receptores sensoriais têm suas capacidades reduzidas, dificultando a percepção aos estímulos traumáticos e agressivos ${ }^{(2)}$. Dessa forma, na presença de fatores mecânicos, a diminuição da sensibilidade atua como fator coadjuvante para integridade de pele prejudicada.

\section{CONCLUSÕES}

Este estudo permitiu identificar incidência de integridade da pele prejudicada em 50\% dos idosos de uma comunidade, com distribuição semelhante entre homens e mulheres, sendo a ocorrência maior na faixa etária de 60 a 69 anos. A característica definidora de maior freqüência foi rompimento da superfície da pele $(85 \%)$. Identificamos fatores relacionados descritos pela NANDA que têm papel coadjuvante no aparecimento das lesões: extremos de idade, fatores imunológicos, alteração do turgor, estado de líquidos alterados, metabolismo alterado, hipertermia, diminuição de sensibilidade e estado nutricional alterado. Os fatores relacionados que tiveram relação causal direta constituíram-se em fatores mecânicos (85\%), exposição 
aumentada à radiação (5\%) e circulação alterada (15\%).

Mediante estes achados, pode-se concluir que este fenômeno merece maior atenção do enfermeiro no atendimento à população de idosos, tendo em vista a menor capacidade dos mesmos com relação aos processos de reparação tecidual. É necessário ainda estudar as intervenções pertinentes para este grupo, no sentido de prevenção e recuperação de lesões, além de promoção da saúde como um todo, que se refletem na higidez do tecido tegumentar.

\section{REFERÊNCIAS}

1- Rodrigues RAP, Diogo MJD. Como cuidar dos idosos. Campinas: Papirus; 1996.

2- Duarte YAO, Diogo MJD. Atendimento domiciliar: um enfoque gerontológico. São Paulo: Atheneu; 2000.

3- Smeltzer SC, Bare BG. Brunner \& Suddarth : tratado de enfermagem médico-cirúrgica. 10ed. Rio de Janeiro: Guanabara Koogan; 2005.
4 Nettina SM. Prática de enfermagem. 7a. ed. Rio de Janeiro: Guanabara Koogan; 2003.

5- 5-Oriá RB, Brito GAC, Ferreira FVA, Santana EM, Fernandes MR. Estudo das alterações relacionadas com a idade na pele humana, utilizando métodos de histomorfometria e autofluorescência. An Bras Dermatol. 2003; 78(4):425-34.

6- North American Nursing Diagnosis Association. Diagnósticos de enfermagem da NANDA: definições e classificação - 2003-2004. Porto Alegre: Artmed; 2005.

7- Norman RA, Bock M. Wound care in geriatrics. Dermatol Ther. 2003;16(3):224-30.

8- Frade MAC, Cursi IB, Andrade FF, Soares SC, Ribeiro WS, Santos SV, Foss NT. Úlcera de perna: um estudo de casos em Juiz de Fora-MG (Brasil) e região. An Bras Dermatol. 2005;80(1):41-6.

9- Costa EFA, Porto CC, Soares AT. Envelhecimento populacional brasileiro e o aprendizado da geriatria e gerontologia. Revista UFG. 2003; 5(2):7-10.

10- Porto CC. Semiologia médica. 5a ed. Rio de Janeiro: Guanabara Koogan; 2005. 